\title{
Memory formation in reversal learning of the honeybee
}

\author{
Ravit Hadar* and Randolf Menzel \\ Neurobiology, Institut für Biologie, Freie Universität Berlin, Berlin, Germany
}

\section{Edited by:}

Jean-Christophe Sandoz, Centre National De La Recherche Scientifique, France

\section{Reviewed by:}

Jean-Marc Devaud, University Paul

Sabatier, France

Nigel E. Raine, Queen Mary University, UK

\section{${ }^{*}$ Correspondence:}

Ravit Hadar, Fachbereich Biologie/ Chemie/Pharmazie, Neurobiologie, Institut für Biologie, Freie Universität Berlin, Königin-Luise-Street 28/30,

14195 Berlin, Germany.

e-mail: ravithadar@hotmail.com

\begin{abstract}
In reversal learning animals are first trained with a differential learning protocol, where they learn to respond to a reinforced odor (CS+) and not to respond to a non-reinforced odor (CS-). Once they respond correctly to this rule, the contingencies of the conditioned stimuli are reversed, and animals learn to adjust their response to the new rule. This study investigated the effect of a protein synthesis inhibitor (emetine) on the memory formed after reversal learning in the honeybee Apis mellifera. Two groups of bees were studied: summer bees and winter bees, each yielded different results. Blocking protein synthesis in summer bees inhibits consolidation of the excitatory learning following reversal learning whereas it blocked the consolidation of the inhibitory learning in winter bees. These findings suggest that excitatory and inhibitory learning may involve different molecular processes in bees, which are seasonally dependent.
\end{abstract}

Keywords: olfactory learning, honey bee, season, protein synthesis inhibitor, reversal learning, memory

\section{INTRODUCTION}

In classical conditioning, animals learn to associate an originally neutral stimulus (CS) with a biologically significant stimulus (US) if the CS is followed by the US (forward pairing). Animals are also capable of acquiring an opposite contingency for a given CS, i.e., the absence of the US. Following Pavlov's (1927) terminology, differential conditioning consists of two such contingencies, where the stimulus which precedes the appearance of the US (CS+) retains an excitatory valence, and the one which predicts the absence of the US (CS-) retains an inhibitory one. In reversal learning the animal is first introduced to differential conditioning and once such discrimination has been learned, the stimuli's contingencies are reversed and the animal learns to adapt its response to the new rule. Following Pavlov (1927), forward pairing of CS with reinforcement generates excitatory learning whereas extinction leads to inhibitory learning. Thus reversal learning is a paradigm entailing rather more complex learning than a simple acquisition and extinction, as the animal has to form such new associations on the background of inverted contingencies. The molecular underpinnings of acquisition and extinction learning are believed to differ, particularly in regard to the requirement of protein synthesis. In a wide range of experimental preparations, protein synthesis inhibition was found to block memory formation of acquisition learning (e.g., Davis and Squire, 1984; Abel et al., 1997; Lattal and Abel, 2001). In extinction on the other hand, the administration of protein synthesis inhibitors yielded conflicting results which probably depend on the experimental protocol used (e.g., Flood et al., 1977; Berman and Dudai, 2001; Stollhoff et al., 2005; Duvarci et al., 2006). Altogether, reversal learning provides an adequate paradigm to study both acquisition and extinction learning and memory.

The honeybee (Apis mellifera) serves as a valid model for the study of the underlying mechanisms of learning and memory (Menzel et al., 2006) for which many paradigms of conditioning were tested. It was found that the results follow the rules of classical conditioning as known from laboratory mammals (Bitterman et al., 1983; Menzel and Bitterman, 1983; Menzel, 1990). Odors are used as CSs, and sucrose solution as US for hungry bees. Several forms of memory developing in series and in parallel have been described leading to lifelong memory under appropriate conditions (Menzel, 1990).

Memory formation has been shown to consist of distinctive phases; each depends on different molecular pathways: short-term and mid-term memories depend on existing proteins, and two forms of long-term memory (LTM) are controlled by different signaling cascades (Menzel and Müller, 1996; Menzel, 1999; Müssig et al., 2010). Notably, early LTM (eLTM) depends on translation and late LTM (ILTM) depends on transcription processes (Wüstenberg et al., 1998; Friedrich et al., 2004). When applied shortly prior to acquisition, emetine, a protein synthesis blocker which inhibits translation processes, is known to inhibit eLTM consolidation in the honeybee. The effects of transcription and translation inhibitors have been studied so far only in simple forward pairing paradigms (Wüstenberg et al., 1998; Menzel et al., 2001; Friedrich et al., 2004) and in extinction paradigms (Stollhoff et al., 2005; Stollhoff and Eisenhardt, 2009).

In the honeybee, reversal learning was found to have a heritable component which is manifested in the rapidity to reverse from the former CS- to the new CS+ association (Ferguson et al., 2001). However, Ben-Shahar et al. (2000) found differences in the extinction rate of the former CS+ during the reversal phase, which were derived from the bees' behavioral state: nurses showed faster rates of extinction than foragers. Taken together, these finding suggest that two dissociable processes constitute the reversal learning, i.e., excitatory learning and inhibitory learning.

Using local anesthetics to block the main output region of the mushroom body (MB), Devaud et al. (2007) were able to demonstrate that the acquisition of reversal learning requires an intact $\mathrm{MB}$ activity, whereas simple differential learning (the first phase in reversal paradigm) was spared. It was also shown that experiencing olfactory reversal learning improves the bee's future performance 
in solving further discrimination reversals (Komischke et al., 2002), a feature that might serve to optimize bee's foraging efficiency when food-source profitability changes. However, those studies were designed so, that the temporal spacing of each phase from the next allowed only the formation of short-term and mid-term memories in this paradigm.

Here the effect of emetine on the eLTM formed after reversal learning was investigated in order to elucidate the consolidation of excitatory and inhibitory associations formed after reversal learning, into eLTM. To this end, each learning phase took place on a different day, when translation-dependent memories are formed. Two groups of honeybees were used: summer bees and winter bees, because it was observed in earlier experiments (Menzel et al., 2001) that inhibiting transcription factors yields different results in summer and winter bees, specifically, winter bees did not develop long-lasting memory following spaced conditioning. We found that blocking protein synthesis during consolidation of reversal learning inhibits the consolidation of the excitatory learning in summer bees whereas consolidation of inhibitory learning was blocked in winter bees.

\section{MATERIALS AND METHODS GENERAL PROCEDURES RELATED TO BEHAVIOR}

The experiments were conducted in Berlin, Germany using honeybees (A. mellifera carnica) from the colonies of the laboratory. Experiments were carried out in summer time (July/August 2009), using bees raised in outdoor hives, and in winter time (November/ December 2009), using bees kept in small flight cages $\left(1 \mathrm{~m}^{3}\right)$ in a glasshouse. One day prior to the experimental procedure, foraging bees were caught at the hive entrance when leaving the hive; they were then immobilized by cooling and harnessed in small metal tubes. In the evening bees were fed to satiation with a 1-M sucrose solution. On each experimental day, bees were fed in the afternoon to satiation and then kept in a dark and humid box at room temperature $\left(\sim 22^{\circ} \mathrm{C}, \sim 70 \%\right.$ humidity $)$.

\section{CONDITIONING OF THE PER}

All acquisition and retrieval trials shared a standardized protocol; each acquisition trial began by positioning a test bee in front of an exhaust fan. Odor stimuli (CS) were applied after $10 \mathrm{~s}$ (duration $4 \mathrm{~s}$ ) and were delivered through $5 \mathrm{ml}$ syringe, each containing a filter paper soaked with $4 \mu \mathrm{L}$ of pure odorant, 2-octanone, and 1-hexanol (Sigma-Aldrich Chemie GmbH). Computer-controlled magnetic valves were used for the delivery of the odorants, allowing constant air flow. The presentation of the US started $3 \mathrm{~s}$ after odor onset by touching the antennae with a toothpick soaked in sucrose solution to induce proboscis extension. US delivery lasted for $4 \mathrm{~s}$ during which animals were allowed to lick sucrose solution with the proboscis (hence $1 \mathrm{~s}$ overlap between CS and US).

On unrewarded trials (CS-) all conditions remained the same, except there was no presentation of the US (sucrose). A positive response was scored if the proboscis was extended during the CS and before the US.

\section{REVERSAL LEARNING PROTOCOL}

On the first day animals were subjected to a differential conditioning protocol with two odorants $\mathrm{A}$ and $\mathrm{B}$ (2-octanone and 1-hexanol), one forward paired with the US (sucrose solution), the other unrewarded (day 1: A+ vs. B-). Each odorant was presented six times in a pseudo-randomized order and the sequence of odor presentation was identical for all subjects (ABBABAABABBA). Odor identities were counter balanced across subjects.

The intertrial interval was $10 \mathrm{~min}$. On the following day the reinforcement pattern was reversed (day 2: A- vs. B+) whereas all other conditions remained constant. Retention tests were carried out on the third day, where both odorants were presented in the absence of reward.

Acquisition curves are presented as percentages of bees showing conditioned PER for each pair of CS+ and CS- presentations, which constitute one block trial.

\section{EMETINE TREATMENT}

Emetine (catalog \#45160; Fluka, Buchs, Switzerland) was dissolved in PBS (in mM: $137 \mathrm{NaCl}, 2.7 \mathrm{KCl}, 10.1 \mathrm{Na}_{2} \mathrm{HPO}_{4}, 1.8 \mathrm{KH}_{2} \mathrm{PO}_{4}, \mathrm{pH}$ 7.2). One microliter of emetine $(10 \mathrm{mM})$ was injected manually into the flight muscle using a calibrated glass capillary. Animals were injected $30 \mathrm{~min}$ before the reversal conditioning. Control bees were injected with $1 \mu \mathrm{l}$ of PBS.

\section{DATA ANALYSIS}

Only animals that survived until the retention test and then showed an unconditioned response to sucrose were included. The ordinates give the probability of PER responses. The McNemar $\chi^{2}$ test (Zar, 1997) was used (SigmaStat) for within-group comparison of the CR to the different odors. The $G$-test for contingency tables (log likelihood ratio) was used when testing the differences in CR for each odor for between group comparisons.

\section{CONTROL EXPERIMENTS}

Control experiments were designed in order to rule out a general effect of emetine on performance. On the first day bees were subjected to a differential conditioning protocol as described above. On the following day, bees were assigned randomly to two groups and were injected with either emetine or PBS, and after $30 \mathrm{~min}$ a retention test for both odors (in the absence of a reward) was carried out. On the third day bees underwent another retention test for both odors.

\section{RESULTS}

\section{SUMMER EXPERIMENTS}

Emetine inhibits the new excitatory learning when applied in summer On the first experimental day, summer bees were trained to differentiate between two odorants, one being rewarded $(\mathrm{A}+)$ whereas the other was presented alone (B-). Each odor was presented six times; by the last differential learning trials the proportions of CRs to the $\mathrm{A}+$ and $\mathrm{B}-$ were 76 and $2 \%$, respectively (McNemar's Test: $\chi^{2}=112.00, p<0.001, \mathrm{df}=1$; Figure 1A).

On the following day, $30 \mathrm{~min}$ prior to reversal learning training, bees were randomly assigned to two groups; one being injected with emetine and the other with PBS (phosphate buffer used as saline for emetine). All bees were then trained to the reversed rule $(\mathrm{A}-\mathrm{vs} . \mathrm{B}+)$. By the last differential learning trials the proportions of CRs to A- and B+ were 4 and $49 \%$ in the PBS group (McNemar's Test: $\chi^{2}=29.03, p=<0.001, \mathrm{df}=1$ ) and 8 and $39 \%$ 
in the emetine group, respectively (McNemar's Test: $\chi^{2}=21.04$, $p=<0.001$, df $=1$; Figure 1B). Emetine injections had no effect on acquisition during this phase.

On the third day, $24 \mathrm{~h}$ after the reversal learning, all bees were subjected to a retention test for both odorants (Figure 1C). The group injected with PBS scored significantly higher for odor B than for odor A (McNemar's Test: $\chi^{2}=4.267, p<0.05$, $\mathrm{df}=1$ ) indicating that the reversal rule had been learned and was remembered. In contrast, the emetine injected group scored the same for both odors (McNemar's Test: $\chi^{2}=0.050, \mathrm{NS}, \mathrm{df}=1$ ), indicating that this group did not remember the reversed rule. Moreover, the emetine injected group scored significantly lower for odor B in comparison with the PBS injected group ( $G$ test: $G=4.254, p<0.05$, $\mathrm{df}=1$ ), which indicates an emetine-treatment induced interference with consolidation of excitatory learning.

\section{Control experiments: when applied $24 \mathrm{~h}$ after differential learning, emetine has no effect on memory retrieval}

On the first experimental day, summer bees underwent a differential conditioning protocol, as described above. By the last differential learning trials the proportions of bees exhibiting the $\mathrm{CR}$ to the $\mathrm{A}+$ and $\mathrm{B}-$ were 74 and $5 \%$, respectively (McNemar's Test: $\chi^{2}=53.01$, $p<0.001, \mathrm{df}=1$; Figure 2A). On the following day, $30 \mathrm{~min}$ prior to a retention test, bees were randomly assigned to two groups; one

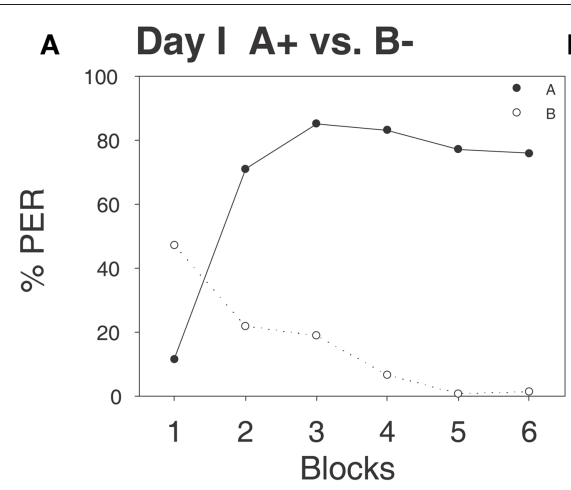

B Day II A- vs. B+

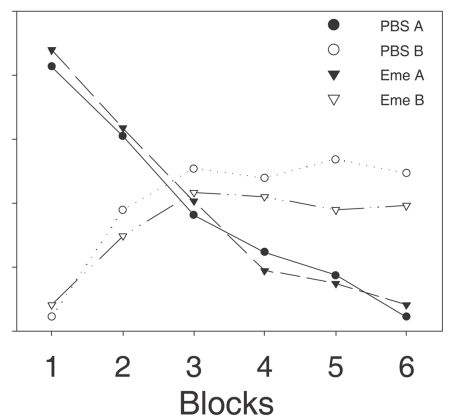

c Day III

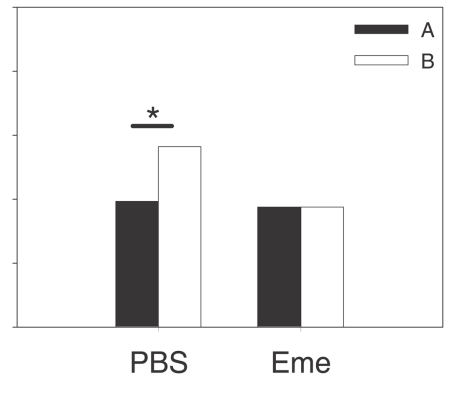

FIGURE 1 | In summer the systemic application of emetine $30 \mathrm{~min}$ before reversal learning inhibits consolidation of the new excitatory learning. Shown are percentages of bees which exhibited proboscis extension responses (PER) evoked by either of the two odorants A (filled shapes and bars) and B (open shapes and bars). (A) On day 1, bees were untreated and trained to the differential conditioning rule (A+vs. B-), each stimulus was presented six times, shown here in six blocks, (solid line and filled circles for A+vs. dashed line and open circles for B-). At the last trials a significant difference in CRs between odors was observed (McNemar's Test, $p<0.005)$. (B) On day 2, 30 min after emetine (triangles) or PBS (circles) injections, a reversal protocol was applied ( $A-v s$. $B+$ ), each stimulus was presented six times, shown here in six blocks, (solid lines for $A+v s$. dashed lines for B-) at the last trials both PBS and emetine groups exhibited a reversed preference (McNemar's Test, $\mathrm{p}<0.005$ for both groups). (C) On day 3 , both groups were subjected to a retention test for both odorants in the absence of a reward. The PBS treated control group showed a significant preference for odor B (McNemar's Test, $N=56, p<0.05$ ), the emetine treated experimental group showed no preference (McNemar's Test, $N=64$, $N S)$ and bees responded significantly less often to odor $B$ than in the PBS group (G test, $\mathrm{p}<0.05$ ).
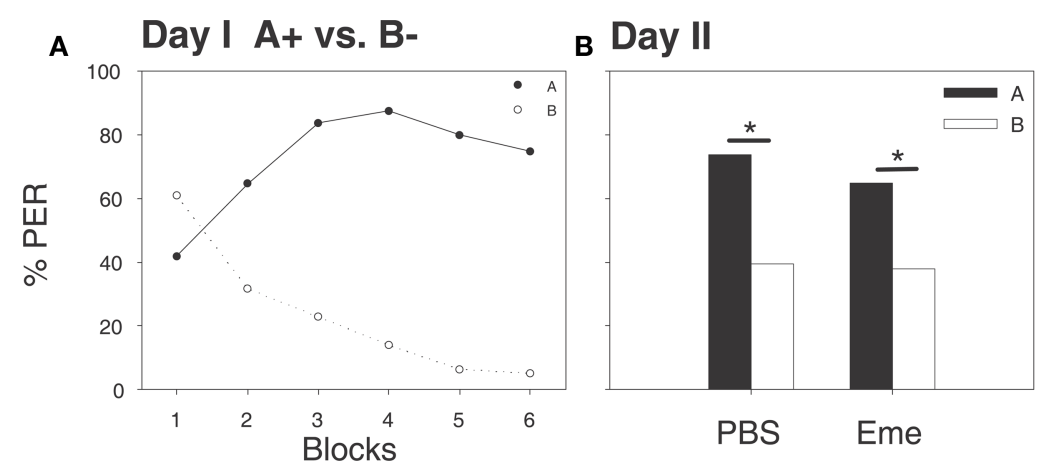

c Day III

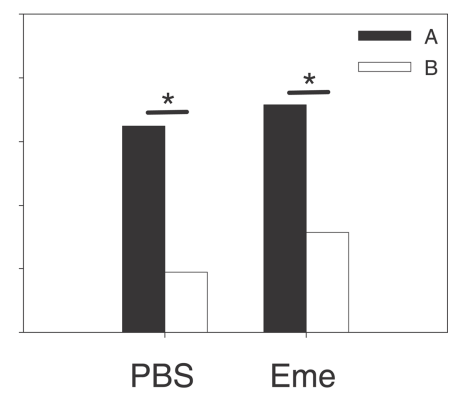

FIGURE 2 |The systemic application of emetine $\mathbf{3 0} \mathrm{min}$ before a retention test has no effect on memory retrieval after $\mathbf{2 4} \mathbf{h}$. Shown are percentages of bees which exhibited proboscis extension responses (PER) evoked by the two odorants $A$ (filled shapes and bars) and B (open shapes and bars). (A) On day 1, bees were untreated and trained to differential conditioning (A+vs. B-) each stimulus was presented six times, shown here in six blocks, (solid line and filled circles for A+vs. dashed line and open circles for B-). For the last trial a significant difference in the percentage of bees exhibiting the CR between odors was observed (McNemar's Test, $p<0.001$ ). (B) On day 2, 30 min after emetine or PBS injections, a retention test was carried out in the absence of reward. Both groups exhibited a significant preference for odor A (McNemar's Test - PBS group: $p<0.05$; Eme group: $p<0.01$ ). (C) On day 3, all groups were subjected to another retention test for both odorants. Both groups scored significantly higher for odor $A$ than for odor B (McNemar's Test - PBS group: $N=37, p<0.05$; Eme group: $N=35, p<0.01$. 
being injected with emetine and the other with PBS. At the retention test both groups scored significantly higher to odor A than to odor $\mathrm{B}$, as shown by their CRs; PBS group 73 and 39\%, respectively (McNemar's Test: $\chi^{2}=8.47, p<0.005$, $\mathrm{df}=1$ ), emetine group 75 and $27 \%$, respectively (McNemar's Test: $\chi^{2}=13.13, p<0.001, \mathrm{df}=1$; Figure 2B). There was no significant difference in the proportions of bees exhibiting a CR between the two experimental groups ( $G$ test odor A: G PBS vs. G Eme $=0.039, \mathrm{NS}, \mathrm{df}=1 ; \mathrm{G}$ test odor B: $\mathrm{G}$ PBS vs. $\mathrm{GEme}=1.31, \mathrm{NS}, \mathrm{df}=1)$. Another retention test was carried out on the third day. Again both groups scored significantly higher for odor A than for odor B, PBS group 64 and 37\%, respectively (McNemar's Test: $\chi^{2}=5.78, p<0.05, \mathrm{df}=1$ ) emetine group 71 and $31 \%$, respectively (McNemar's Test: $\chi^{2}=7.68, p<0.01, \mathrm{df}=1$; Figure 2C). Again both groups did not differ in their proportion of bees exhibiting a CR to both odorants ( $G$ test odor A: G PBS vs. G Eme $=0.035, \mathrm{NS}, \mathrm{df}=1 ; G$ test odor B: G PBS vs. G Eme $=0.032$, $\mathrm{NS}, \mathrm{df}=1$ ). A general non-specific effect of emetine on learning and memory can thus be ruled out.

\section{WINTER EXPERIMENTS}

\section{Emetine inhibits the new inhibitory learning when applied in winter}

The same protocol was applied to winter bees (Figure 3). By the last differential learning trials on day 1 the proportions of bees exhibiting the $\mathrm{CR}$ to the $\mathrm{A}+$ and $\mathrm{B}-$ were 63 and 7\%, respectively (McNemar's Test: $\chi^{2}=68.01, p=<0.001$, df $=1$; Figure 3A). On the following day, 30 min prior to reversal learning training, bees were randomly assigned to two groups; one being injected with emetine and the other with PBS (phosphate buffer used as saline for emetine). All bees were then trained the reversed rule (A-vs. B+). By the last differential learning trials the proportions of bees exhibiting the CR to the A- and B+ were 22 and $57 \%$ in the PBS group (McNemar's Test: $\chi^{2}=14.06, p=<0.001, \mathrm{df}=1$ ), respectively, and 25 and $61 \%$ in the emetine group (McNemar's Test: $\chi^{2}=17.05, p=<0.001$, df $=1$ ), respectively (Figure 3B).
As in the summer experiments, emetine injections had no effect on the acquisition curves during this phase. On the third day a retention test for both odorants was carried out (Figure 3C). As in summer bees, the group injected with PBS scored significantly higher for odor B than for odor A (McNemar's Test: $\chi^{2}=9.091$, $p<0.05, \mathrm{df}=1$ ) indicating that bees learned to associate odor $B$ with reward. As in summer, no significant difference between the two odorants was observed in the emetine injected group (McNemar's Test: $\chi^{2}=0.571, \mathrm{NS}, \mathrm{df}=1$ ). However, as opposed to the results achieved in the summer where emetine inhibited the excitatory association, here the emetine injected group scored significantly higher for odor A, when compared with the PBS group ( $G$ test: $G=4.0422, p<0.05, \mathrm{df}=1$ ).

\section{Control experiments: when applied $24 \mathrm{~h}$ after differential conditioning, emetine has no effect on memory retrieval}

On the first experimental day, winter bees underwent a differential conditioning protocol, as described above. By the last differential learning trials the proportions of bees exhibiting CRs to the A+ and B- were 65 and $15 \%$, respectively (McNemar's Test: $\chi^{2}=27.57$, $p<0.001, \mathrm{df}=1$; Figure 4A). On the following day, $30 \mathrm{~min}$ prior to a retention test, bees were randomly assigned to two groups; one being injected with emetine and the other with PBS. At the retention test both groups scored significantly higher to odor A than to odor B; PBS group 64 and 38\%, respectively (McNemar's Test: $\chi^{2}=4.26, p<0.05, \mathrm{df}=1$ ), emetine group 74 and $42 \%$, respectively (McNemar's Test: $\chi^{2}=4.76, p<0.05$, df $=1$; Figure 4B). There was no significant difference in the proportions of bee exhibiting the CR between the two experimental groups ( $G$ test odor A: PBS vs. Eme $=0.74, \mathrm{NS}, \mathrm{df}=1 ; G$ test odor B: PBS vs. Eme $=0.15$, $\mathrm{NS}, \mathrm{df}=1)$. Another retention test was carried out on the third day, again, both groups scored significantly higher for odor A than for odor B, PBS group 70 and 41\%, respectively (McNemar's Test: $\chi^{2}=5.06 ; p<0.05 ; \mathrm{df}=1$ ) emetine group 73 and $38 \%$, respectively

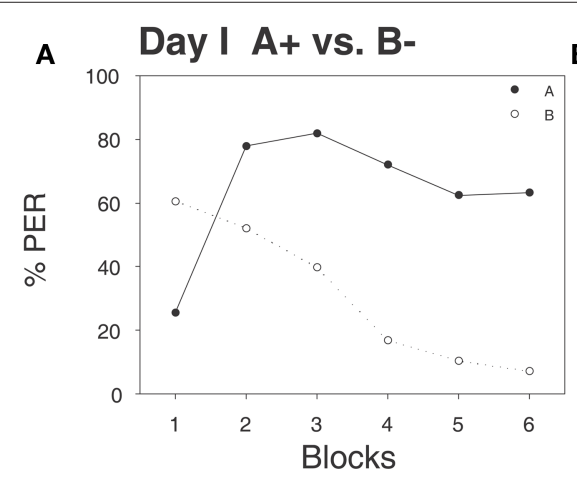

FIGURE 3 | In winter the systemic application of emetine $\mathbf{3 0} \mathrm{min}$ before reversal learning inhibits consolidation of the new inhibitory learning. Shown are percentages of bees which exhibited proboscis extension responses (PER) evoked by the two odorants A (filled shapes and bars) and B (open shapes and bars). (A) On day 1 , bees were untreated and differentially conditioned ( $A+$ vs. B-) each stimulus was presented six times, shown here in six blocks, (solid line and filled circles for A+ vs. dashed line and open circles for B-). At the last trials a significant difference in CRs between odors was observed (McNemar's Test, $p<0.005)$. (B) On day 2, 30 min after emetine (triangles) or PBS (circles)
B Day II A- vs. B+

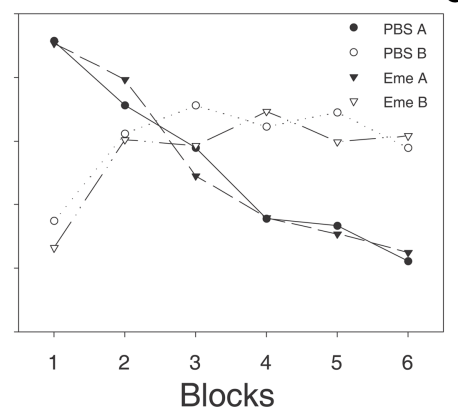

c Day III

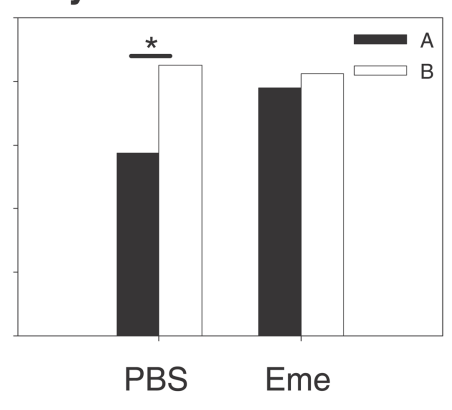

injections, a reversal protocol was applied ( $A-v s . B+$ ), each stimulus was presented six times, shown here in six blocks, (solid lines for A+ vs. dashed lines for B-), at the last trials both PBS and emetine groups exhibited a reversed preference (McNemar's Test, $p<0.05$ for both groups). (C) On day 3, all groups were subjected to retention tests for both odorants in the absence of a reward. The PBS group showed a significant preference for odor B (McNemar's Test, $N=40, p<0.05$ ), the emetine group showed no preference (McNemar's Test, NS, $N=45$ ) and scored significantly higher for odor A than PBS group ( $G$ test, $p<0.05)$. 


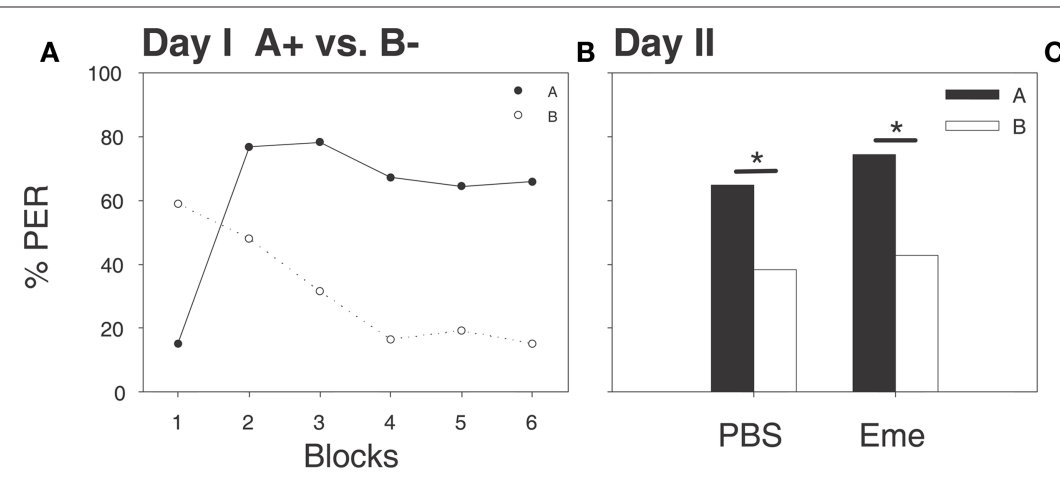

c Day III

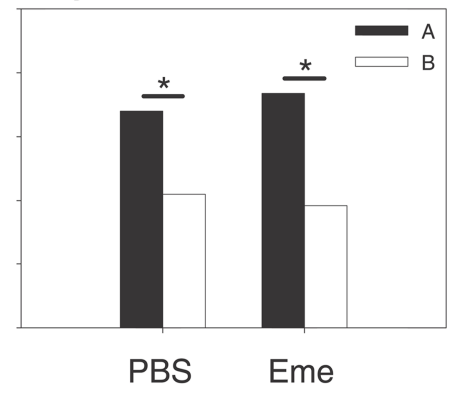

FIGURE 4 |The systemic application of emetine $\mathbf{3 0} \mathrm{min}$ before a retention test has no effect on memory retrieval after $\mathbf{2 4} \mathbf{h}$. Shown are percentages of bees which exhibited proboscis extension responses (PER) evoked by the two odorants A (filled shapes and bars) and B (open shapes and bars). (A) On day 1 , bees were untreated and differentially conditioned ( $A+v s$. B-) each stimulus was presented six times, shown here in six blocks, (solid line and filled circles for A+ vs. dashed line and open circles for B-). At the last trials a significant difference in CRs between odors was observed (McNemar's Test, $p<0.001$ ). (B) On day 2, 30 min after emetine or PBS injections, a retention test was carried out in the absence of reward; both groups exhibited a significant preference for odor A (McNemar's Test: PBS group: $N=31, p<0.05$; Eme group $N=34$, $p<0.01$ ). (C) On day 3, all groups were subjected to another retention test for both odorants. Both groups scored significantly higher for odor $\mathrm{A}$ than for odor $\mathrm{B}$ (McNemar's Test: PBS group: $p<0.05$; Eme group: $p<0.01$ ).
(McNemar's Test: $\chi^{2}=6.05, p<0.05, \mathrm{df}=1$; Figure 4C). Again both groups did not differ in the proportion of bees exhibiting the CR to both odorants ( $G$ test odor A: G PBS vs. G Eme $=0.05, \mathrm{NS}, \mathrm{df}=1$; $G$ test odor B: G PBS vs. G Eme $=0.09, \mathrm{NS}, \mathrm{df}=1)$. As in summer, a general non-specific effect of emetine on learning and memory can thus be ruled out.

\section{DISCUSSION}

Two learning processes take place while an animal experiences a reversed CS-US contingency: a new excitatory learning and a new extinction learning of the original memory. Unlike a regular extinction, reversal learning involves the continued delivery of a reinforcer and a manifestation of a new preference is hence formed. It has been long known that new memories must be stabilized if they are to persist; this process is called consolidation and requires a cascade of intracellular events (McGaugh, 2000; Dudai, 2004).

The administration of amnestic agents during a discrete time window following learning can disturb the formation of long-term memories. In the honeybee, the systemic administration of emetine, a translation inhibitor, shortly before an absolute appetitive conditioning yields no effect on the learning process but blocks consolidation of long-term memory when tested at $24 \mathrm{~h}$ after acquisition (Stollhoff et al., 2005).

The effect of protein synthesis inhibitors on memory formation has been investigated so far in honeybees in either simple forward conditioning, or in regular extinction paradigms (Wüstenberg et al., 1998; Friedrich et al., 2004; Stollhoff et al., 2005). In addition, eLTM is affected by actinomycin D, a transcription-inhibitor, under spaced conditioning but not under massed conditioning (Menzel et al., 2001). Under a regular extinction paradigm, the emetine effect depends on the number of retrieval trials presented. When applied systematically $30 \mathrm{~min}$ before the presentation of two retrieval trials (non-rewarded CS presentations), it blocks the extinction learning at a 24-h retention test, whereas for five retrieval trials the spontaneous recovery at $24 \mathrm{~h}$ retention test is blocked (Stollhoff et al., 2005).
The present study tested the different effects of protein synthesis inhibitor on reversal learning in two groups of honeybees, summer and winter bees.

The main findings from these experiments are that the requirements for protein synthesis in winter bees and summer bees appear to differ with respect to the kind of memory consolidation. In general, emetine did not fully block reversal learning in either summer bees, or in winter bees. In summer bees emetine injected shortly before reversal learning impaired the manifestation of the new CS-US relation but did not affect the extinction of the original preference, when tested $24 \mathrm{~h}$ later. In the winter bees however, emetine yielded an inverse effect: the manifestation of the new CS-US relation remained intact, whereas the extinction of the original preference was blocked, when tested $24 \mathrm{~h}$ later. These results suggest a double dissociation with respect to the protein synthesis requirements in reversal learning: emetine targets different memories (excitatory memory vs. inhibitory memory), and this effect is different with respect to the line of bees used (summer vs. winter).

It has already been suggested that seasonal variations in honeybees might result in a range of changes from behavior over neurotransmitter and pheromones levels to protein metabolism (Crailsheim, 1986; Currie and Jay, 1988; Harris and Woodring, 1992; Balderrama et al., 1996). Winter bees used in this study were kept under rather artificial conditions. They were housed in small flight cages under circadian illumination, humidity, and temperature conditions that mimicked summer. The bees were foraging for sucrose and pollen, and the colony did not form a winter cluster. The queen continued or started to lay eggs at a low rate. It was observed that in contrast to summer bees, these bees did not form transcription-dependent lLTM after multiple spaced conditioning trials (Menzel et al., 2001). Thus the hormonal status of winter bees that are exposed to simulated summer conditions must be different from real summer bees. So far it has been believed that these differences affect consolidation of ILTM but our study shows that they also affect consolidation of translation-dependent eLTM 
in a learning-dependent way. In the future it would be interesting to investigate the effects of transcription inhibitors on the ILTM of reversal learning.

Translation-dependent memory consolidation requires existing mRNA and a mechanism that targets the synthesized proteins to the respective synaptic sites. Our findings suggest that the excitatory and inhibitory memory traces after olfactory reversal conditioning are differently dependent on cellular mechanisms that express the seasonal hormonal changes. In Drosophila the short-term memory trace of excitatory aversive conditioning and that of extinction learning of such excitatory learning (thus a form of inhibitory learning) appear to depend on different molecular mechanisms of the same neurons, the gamma lobe Kenyon cells of the MB (Schwärzel et al., 2002). It is also known in Drosophila that the transition from short-term to long-term olfactory aversive memory is accompanied by a shift from gamma lobe related Kenyon cells of the MB to vertical lobe related cells (Pascual and Preat, 2001). It is not known, however, whether the consolidation of such excitatory and inhibitory memory traces in Drosophila involves only translation or both transcription and translation. We also do not know yet for the bee whether the effects we see may also require transcription. Recently, using a series of single-gene Drosophila mutants, Qin and Dubnau (2010) found that extinction of olfactory aversive 1 day memory depends on different molecular mechanisms than those involved in associative learning. Other supporting evidence for the dissociation between classical learning and extinction learning arise from vertebrate studies in which pharmacological and genetics disruptions were shown to affect extinction but not classical conditioning (e.g., Cain et al., 2002; Marsicano et al., 2002). Taken together, we interpret our data as supporting the concept developed for Drosophila with respect to the mechanistic

\section{REFERENCES}

Abel, T., Nguyen, P. V., Barad, M., Deuel, T. A., Kandel, E. R., and Bourtchouladze, R. (1997). Genetic demonstration of a role for PKA in the late phase of LTP and in hippocampus-based long-term memory. Cell 88, 615-626.

Balderrama, N., Núñez, J., Giurfa, M., Torrealba, J., De Albornoz, E. G., and Almeida, L. O. (1996). A deterrent response in honeybee (Apis mellifera) foragers: dependence on disturbance and season. J. Insect Physiol. 42, 463-470.

Ben-Shahar, Y., Thompson, C. K., Hartz, S. M., Smith, B. H., and Robinson, G. E. (2000). Differences in performance on a reversal learning test and division of labor in honey bee colonies. Anim. Cogn. 3, 119-125.

Berman, D. E., and Dudai, Y. (2001). Memory extinction, learning anew, and learning the new: dissociations in the molecular machinery of learning in cortex. Science 291, 2417-2419.
Bitterman, M. E., Menzel, R., Fietz, A., and Schaefer, S. (1983). Classical conditioning of proboscis extension in honeybees (Apis mellifera). J. Comp. Psychol. 97, 107-119.

Cain, C. K., Blouin, A. M., and Barad, M. (2002). L-type voltage-gated calcium channels are required for extinction, but not for acquisition or expression, of conditional fear in mice. J. Neurosci. 22, 9113-9121.

Crailsheim, K. (1986). Dependence of protein metabolism on age and season in the honeybee (Apis mellifica carnica pollm). J. Insect Physiol. 32, 629-634.

Currie, R. W., and Jay, S. C. (1988). The influence of a colony's state, time of year and drifting behaviour on the acceptance and longevity of adult drone honeybees (Apis mellifera L.). J. Apic. Res. 27, 219-226.

Davis, H. P., and Squire, L. R. (1984). Protein synthesis and memory: a review. Psychol. Bull. 96, 518-559.

Devaud, J.-M., Blunk, A., Podufall, J., Giurfa, M., and Grünewald, B. (2007). Using local anaesthetics to block and molecular separations between excitatory and inhibitory memory traces. If the transition from short to long-term memory would lead to a separation at the network level, a specific control by hormonal factors expressing the differences between summer and winter bees may be more easily understood. In such a scenario the transfer of excitatory and inhibitory memory traces to the specific networks for long-term memory store would be differently controlled by these hormonal factors.

In this study, differences between summer and winter bees are also evident in the acquisition curves and the retention tests of the reversal learning, irrespective of the experimental groups. Summer bees display general lower levels of proboscis extension response during the acquisition of the reversal learning, compared to winter bees. This also holds true for the retention test on the third day. Such a disparity might result from different brain levels of the biogenic amine octopamine, which is known to influence response threshold to sucrose (Page and Erber, 2002), and its brain levels are correlated both with age and behavioral specialization of bees (Schulz and Robinson, 1999; Wagener-Hulme et al., 1999). In addition, injections of octopamine to specific brain regions served as a substitute for sucrose in an associative learning (Hammer and Menzel, 1998), again pointing to its involvement in the processing of sucrose reward.

\section{ACKNOWLEDGMENTS}

The authors would like to thank Dorothea Eisenhardt for her fruitful discussions and helpful comments. Johannes Felsenberg is gratefully acknowledged for his valuable support during the experiments. We thank the Academic Editor and the reviewers for their fruitful comments. The first author also acknowledges support from the Deutscher Akademischer Austauschdienst (DAAD) for providing a doctoral scholarship grant. neuronal activity and map specific learning tasks to the mushroom bodies of an insect brain. Eur. J. Neurosci. 26, 3193-3206.

Dudai, Y. (2004). The neurobiology of consolidations, or, how stable is the engram? Annu. Rev. Psychol. 55, 51-86.

Duvarci, S., Mamou, C. B., and Nader, K. (2006). Extinction is not a sufficient condition to prevent fear memories from undergoing reconsolidation in the basolateral amygdale. Neuroscience 24, 249-260.

Ferguson, H. J., Cobey, S., and Smith, B. H. (2001).Sensitivity to a change in reward is heritable in the honeybee, Apis mellifera. Anim. Behav. 61, 527-532.

Flood, J. F., Jarvik, M. E., Bennett, E. L., Orme, A. E., and Rosenzweig, M. R. (1977). Protein synthesis inhibition and memory for pole jump active avoidance and extinction. Pharmacol. Biochem. Behav. 7, 71-77.

Friedrich, A., Thomas, U., and Muller, U. (2004). Learning at different satiation levels reveals parallel functions for the
cAMP-protein kinase A cascade in formation of long-term memory. J. Neurosci. 2, 4460-4468.

Hammer, M., and Menzel, R. (1998). Multiple sites of associative odor learning as revealed by local brain microinjections of octopamine in honeybees. Learn. Mem. 5, 146-156.

Harris, J. W., and Woodring, J. (1992). Effects of stress, age, season, and source colony on levels of octopamine, dopamine and serotonin in the honeybee (Apis mellifera L.) brain. J. Insect Physiol. 38, 29-35.

Komischke, B., Giurfa, M., Lachnit, H., and Malun, D. (2002). Successive olfactory reversal learning in honeybees. Learn. Mem. 9, 122-129.

Lattal,K.M., and Abel, T. (2001). Different requirements for protein synthesis in acquisition and extinction of spatial preferences and context-evoked fear. J. Neurosci. 21, 5773-5780.

Marsicano, G., Wotjak, C. T., Azad, S. C., Bisogno, T., Rammes, G., Cascio, M. G., Hermann, H., Tang, J., Hofmann, C., Zieglgänsberger, W., Di Marzo, V., 
and Lutz, B. (2002). The endogenous cannabinoid system controls extinction of aversive memories. Nature 418 , 530-534.

McGaugh, J. L. (2000). Memory: a century of consolidation. Science 287, 248-251.

Menzel, R. (1990). "Learning, memory, and 'cognition' in honeybees," in Neurobiology of Comparative Cognition, eds R. P. Kesner and D. S. Oltenpp (Hillsdale, NJ: Erlbaum), 237-292.

Menzel, R. (1999). Memory dynamics in the honeybee. J. Comp. Physiol. 185, 323-340.

Menzel, R., and Bitterman, M. E. (1983). "Learning by honeybees in an unnatural situation," in Behavioral Physiology and Neuroethology: Roots and Growing Points, eds E. Huber and H. Markl (Berlin: Springer), 206-215.

Menzel, R., Leboulle, G., and Eisenhardt, D. (2006). Small brains, bright minds. Cell 124, 237-239.

Menzel, R., Manz, G., Menzel, R. M., and Greggers, U.(2001). Massed and spaced learning in honey-bees: the role of CS, US, the inter-trial interval and the test interval. Learn. Mem. 8, 198-208.
Menzel, R., and Müller, U. (1996). Learning and memory in honeybees: from behavior to neural substrates. Annu. Rev. Neurosci. 19, 379-404.

Müssig, L., Richlitzki, A., Rössler, R., Eisenhardt, D., Menzel, R., and Leboulle, G. (2010). Acute disruption of the NMDA receptor subunit NR1 in the honeybee brain selectively impairs memory formation. J. Neurosci. 30, 7817-7825.

Page, R. E. Jr., and Erber, J. (2002). Levels of behavioral organization and the evolution of division of labor. Naturwissenschaften 89, 91-106.

Pascual, A., and Preat, T. (2001). Localization of long-term memory within the Drosophila mushroom body. Science 294, 1115-1117.

Pavlov, I.P. (1927).Lectures on Conditioned Reflexes. New York: International Publishers.

Qin, H., and Dubnau, J. (2010). Genetic disruptions of Drosophila Pavlovian learning leave extinction learning intact. Genes Brain Behav. 9, 203-212.

Schulz, D. J., and Robinson, G. E. (1999). Biogenic amines and division of labor in honey bee colonies: behaviorally related changes in the antennal lobes and agerelated changes in the mushroom bodies. J. Comp. Physiol. A 184, 481-488.

Schwärzel, M., Heisenberg, M., and Zars, T. (2002). Extinction antagonizes olfactory memory at the subcellular level. Neuron 35, 951-960.

Stollhoff, N., and Eisenhardt, D. (2009). Consolidation of an extinction memory depends on the unconditioned stimulus magnitude previously experienced during training. J. Neurosci. 29 , 9644-9650.

Stollhoff, N., Menzel, R., and Eisenhardt, D. (2005). Spontaneous recovery from extinction depends on the reconsolidation of the acquisition memory in an appetitive learning paradigm in the honeybee (Apis mellifera). J. Neurosci. 25, 4485-4492.

Wagener-Hulme, C., Kuehn, J. C., Schulz, D. J., and Robinson, G. E. (1999). Biogenic amines and division of labor in honey bee colonies. J. Comp. Physiol. A 184, 471-479.

Wüstenberg, D., Gerber, B., and Menzel, R. (1998).Short communication: long but notmedium-term retention of olfactory memories in honeybees is impaired by actinomycin D and anisomycin. Eur. J. Neurosci. 10, 2742-2745.

Zar, J. H. (1997). Biostatistical Analysis. Englewood Cliffs, NJ: Prentice Hall.

Conflict of Interest Statement: The authors declare that the research was conducted in the absence of any commercial or financial relationships that could be construed as a potential conflict of interest.

Received: 29 June 2010; accepted: 25 November 2010; published online: 13 December 2010.

Citation: Hadar R and Menzel R (2010) Memory formation in reversal learning of the honeybee. Front. Behav. Neurosci. 4:186. doi: 10.3389/fnbeh.2010.00186

Copyright (c) 2010 Hadar and Menzel. This is an open-access article subject to an exclusive license agreement between the authors and the Frontiers Research Foundation, which permits unrestricted use, distribution, and reproduction in any medium, provided the original authors and source are credited. 